\title{
The Newsvendor Problem Based on Multiple Reference Points
}

\author{
Sen Zhou \\ School of Management, Jinan University, Guangzhou, China \\ Email:2747170773@qq.com
}

How to cite this paper: Zhou, S. (2016) The Newsvendor Problem Based on Multiple Reference Points. Open Journal of Sociai Sciences, 4, 227-240.

http://dx.doi.org/10.4236/jss.2016.49019

Received: August 26, 2016

Accepted: September 26, 2016

Published: September 29, 2016

Copyright $\odot 2016$ by author and Scientific Research Publishing Inc. This work is licensed under the Creative Commons Attribution International License (CC BY 4.0).

http://creativecommons.org/licenses/by/4.0/

(c) (i) Open Access

\begin{abstract}
This article will introduce the minimum requirement and the statue quo reference points into newsvendor model. Then the problem of decision bias can be well explained through researching multiple reference points. Many papers confirm the loss aversion is one of the important factors in the decision-making bias and plays an important role in ordering decisions. We propose crossing failure is more important than the loss aversion depending on the decision maker's degree of the aversion. In this note, we present situation where minimum requirement and status quo reference point impact the order decision and give a breakthrough in the study of multiple reference points of newsvendor problem.
\end{abstract}

\section{Keywords}

Minimum Requirement, Statue Quo, Multiple Reference Points, Newsvendor Model, Decision Bias

\section{Introduction}

The traditional newsvendor model is a basic model to analysis inventory problems [1]. In the stochastic demand, the newsvendor can get the information about optimal order quantity from the profit maximization. It helps the newsvendor to balance cost of ordering too much against cost of ordering too few. Later, scholars make a lot of extension in the traditional newsvendor model such as dealing with different objectives and utility functions, pricing policies, discounting structures and different states of information about demand (Khouja 1999 [2]).

Although we can get the information about optimal order quantity, the real order decision is inconsistent with optimal order quantity under rational conditions. Eeckhoudt et al. (1995) [3] show that a risk-averse newsvendor would order fewer quantity comparing to a risk-averse newsvendor. Agrawal and Seshadri (2000) [4] find that a 
risk-averse newsvendor would order more or less items with price affecting the demand distribution. Through newsvendor experiments, Schweitzer and Cachon (2000) [5] identified the existence of pull-to-center effect which revealed decision bias in high or low profit setting. For the decision bias, Wang and Webster (2009) [6] use a loss aversion model to illustrate this phenomenon. Two major factors, overage loss and underage loss in his article, influence newsvendor's order decision.

Under expected utility theory [7] [8] framework, decision bias can not be explained in traditional newsvendor model. However, the development of Prospect Theory [9] [10] provides us with assistance. In recent years, many scholars turned to the study of the reference point. The newsvendor problem based on status quo reference point [6] solves part of problems of decision bias. However, the research of multiple reference point gives a larger concept which can be applied to analyze decision bias. Wang and Johnson (2012) [11] show a tri-reference point theory which summarize three reference point, MR, SQ and G (MR refers to minimum requirement; SQ refers to status quo; G refers to goal). Kacelnik [12] reveals the importance of minimum requirement reference point. Wang and Johnson (2012) [11] further propose that the most important psychological preference is MR reference. As far as we know, Wang and Webster [6] use a conception of loss-aversion to illustrate decision bias. Their model is based on the status quo reference point. However, zero profit SQ reference point is not the only factor. And the effect of crossing failure is much more important than the effect of loss aversion in people's psychological. Considering a single reference point is no longer the only direction. People would premeditate multiple reference point, e.g., Diener (2009) [13] holds people will compare decision results with different reference points, and Ordonez, Connolly et al. (2000) [14] propose two reference points impact on decision making. We extend loss-aversion model by considering MR and SQ reference points and summarize comparative statics of price and cost changes.

Our main aim is to introduce multi-reference point newsvendor model that analyze order decision bias. The newsvendor model based on MR and SQ reference points extends the conception of loss aversion which further elaborates how crossing failure and loss aversion cause the decision bias in newsvendor problem of multi-reference point. Through a series of analysis, we summarize: 1) Considering MR and SQ reference points, the newsvendor will order less than the risk-neutral newsvendor; 2) If the parameter of failure is big enough, the loss of a sense of failure is much more intense than the loss of a sense of loss; 3) As with traditional concepts, the newsvendor's optimal order quantity will increase in retail price and decrease in wholesale price; 4) The quantity decreased while there exist MR reference point. And with MR increasing, the newsvendor will decrease optimal order quantity; 5) The bigger the value of the aversion parameter is, the greater the newsvendor decrease.

The contributions of our research are showed from three aspects. First, on the basis of experiment and theory, we introduce multi-reference point theory of behavioral science into newsvendor model so that we can further quantify the problem of order decision bias. Second, we set up newsvendor model based on MR and SQ reference 
points. And by discussing about the influence of retail price, wholesale price, our research shows why crossing failure is more important than loss aversion. Then we expand our perspective to the supply chain inefficiency and uncoordinated and put forward some new policies responding to this effect.

The following part is organized like this: In Section 2, we briefly review the related newsvendor and reference point literature. In Section 3, we analyze the basic model. In Section 4, we analyze the corresponding parameters. In Section 5, we draw our conclusions and provide some suggestions for future research.

\section{Related Newsvendor Literature}

Our decision-making behavior is affected by the reference point. With two experiments, Rosch (1975) [15] proves that the result is affected by the reference point. In the experiment papers about newsvendor problem and reference point, Schweitzer and Cachon (2000) propose an important perception of decision bias. Su (2008) [16] verify the newsvendor's irrational behavior. Eeckhoudt et al. (1995) [3], Agrawal and Seshadri (2000) [4] also identify order items inconsistent with traditional rational decision in practice. Recently, many researchers attempt to analyze the bias through reference point. Like Mahesh Nagarajan and Steven Shechter (2013) [17] combine the newsvendor model with prospect theory, but ignore the existence of reference point, Xiaobo Zhao and Wei Geng (2014) [18] propose Mahesh Nagarajan and Steven Shechter's article does not set reference point, and set the middle of profits as a reference point, proposing different parameters can get different results. BhavaniShankerUppari and Sameer Hasija (2014) [19] set the mean demand of the corresponding profit as a reference point, because the subjects often anchor in the average place. However, their reference points are not random, only using a special point as a reference point. Charles X. Wang and Scott Webster (2009) show us a newsvendor model of a zero profit statue quo reference point. Their research only consider a single SQ reference point. Based on Prospect Theory, adding reference point to newsvendor model is a hot research direction to analyze ordering decision bias.

Research, under conditions of risk and uncertainty in decision-making, has changed a lot. in 1944 Vonnuemna and Mogrnestern [8] put forward expected utility theory from a rational point of view. However, the theory does not explain the emergence of a lot of people's behavior in vision. "Allais Paradox" (1953) [20] presented the results of the people's over-emphasis on certainty results. "Ellsberg Paradox" (1961) [21] showed the situation of objective probability inconsistent with people's subjective probability. Kahneman and Tversky (1979) summarized the paradoxs above and proposed Prospect Theory. Later he improved the discussion about probability so that extending Prospect Theory to Cumulative Prospect Theory (1992). In 2008, sehmidt and starmer [22] presente the third generation of Prospect Theory. Focus on the minimum requirement, status quo and goal reference points, Wang and Johnson set forth a tri-reference point theory of decision making under risk. On the mechanism of the current reference points, there are two explanations: "Norm theory" and "Regret Theory". Kahneman 
and Miller believe the reference point can be either a priori, it can be built after the event for the results. The program which is not selected as the reference point form the basis for an individual assessment of the decision-making results [23]. "Regret Theory" emphasizes there is a choice because there is no other program which led to the "regret function". If the result is better than the other possible outcomes, the individual will produce a sense of joy, otherwise will regret it [24].

Kahneman and Tversky (1979) and other papers all emphasize the importance of status quo reference point and ignore other reference points. Yate and Stone (1992) [25] classify a variety of reference points. One is status quo reference point, the other non-status quo reference point. Wang and Johnson propose the most important reference point is minimum requirement reference point. Crossing failure is far more important than avoiding loss. Kacelnik \& Bateson (1997) [12], Real \& Caraco (1986) [26], and Stephens \& Krebs (1986) [23] show minimum requirements for risk appetite in animal feeding studies has an important role. In the research on negotiation. Diener (2009) [13] holds people will compare decision results with different reference points.

However, the theoretical analysis of double reference points is seldom researched. while the research based on multi-reference point is researched in some economic and management field, like Sullivan and Kida (1995) [24] propose multiple points of reference are important determinants of risky behavior in many decision contexts through situational experiments, we cannot explain the mechanism of multiple points of reference in the stochastic demand.

\section{Newsvendor Model Based on the $M R$ and the $S Q$ of Reference Points}

Under uncertainty, a newsvendor makes decision through selling short-life-cycle products. For the newsvendor problem, we assume that the acquisition cost from a supplier is $w$ per unit and retail price is $p$ per unit $(p>w)$. We also assume that there is no shortage cost and salvage value. Demand $x$, a random variable at the time of purchasing from the supplier, is characterized by a probability density function $f(x)$ and a cummunicative distribution function $F(x)$. Then we can get the profit function. We can get the profit function in Equation (1).

$$
\pi(x, Q)=\pi=\left\{\begin{array}{lc}
\pi_{+}=p x-w Q, & 0 \leq x<Q \\
\pi_{-}=(p+s-w) Q-s x, & Q \leq x \leq \sup I
\end{array}\right.
$$

The plot of them are shown in Figure 1.

Where $w \in(0,1)$ and $p>w$. We define $M R$ as the value of minimum requirement reference point with psychological value parameter $\beta_{F}$ and $S Q$ as the value of statue quo reference point with psychological value parameter $\beta_{-}$. They are both exogenous. Order quantity $q_{M}(Q)$ responds to $M R$. Order quantity $q_{S}(Q)$ responds to $S Q$. Let $q_{S}(Q)=\frac{w Q}{p}$ and $q_{M}(Q)=\frac{w Q+M R}{p}$.

Lemma 1. For any $Q \in I$, if $M R \geq-w Q$, then the newsvendor has three breakeven 


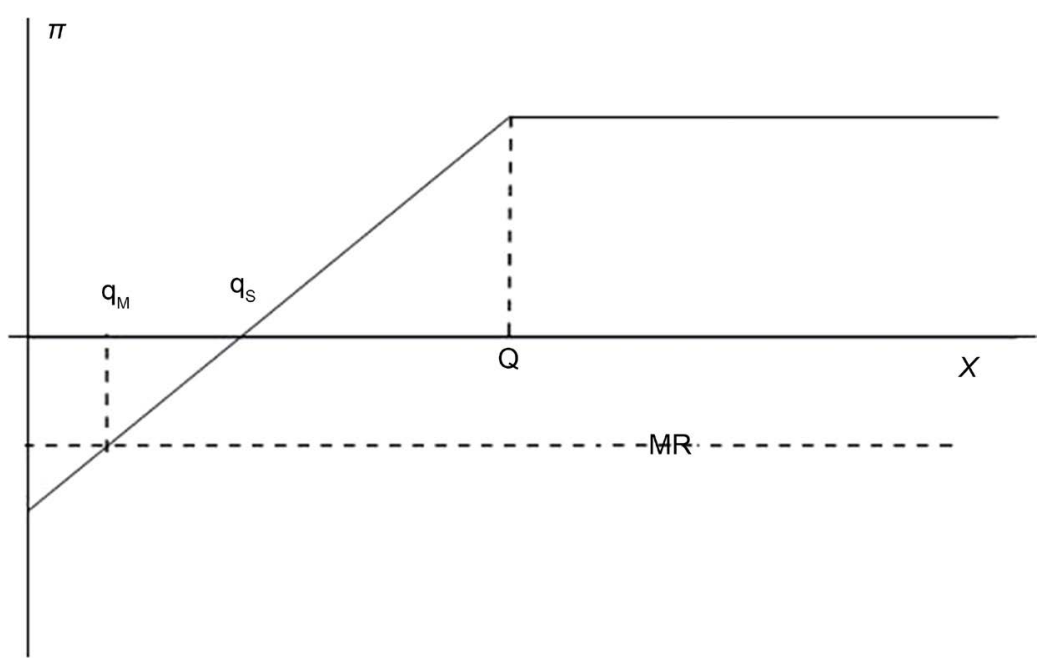

Figure 1. The profit function.

quantities of realized demand in view of MR and SQ reference points, $q_{S}(Q)$ and $q_{M}(Q)$ where if $x<q_{M}(Q)$, the newsvendor's realized profit belongs to failure part and is negative, if $q_{M}(Q)<x<q_{S}(Q)$, the newsvendor's realized profit belongs to loss part and is also negative, and if $q_{S}(Q)<x$, the newsvendor's realized profit is positive.

Lemma 1 explain the influence of mechanism of $M R$ and $S Q$ reference point. If realized demand $x$ is too low, i.e., $x<q_{S}(Q)$, then the newsvendor will get a loss. Whether this effect that making the profit achieving $M R$ depends on the level of ordering cost $w$. When the ordering cost $w$ is high i.e., $w \geq \frac{-M R}{Q}$, the realized demand makes the profit achieving $M R$. Under the MR reference point, the newsvendor will feel failure. Above the $S Q$ reference point, the newsvendor will obtain more benefits. We normalize this to 1 .

In ordering decision-making research literature, loss-aversion and failure-avoiding and target-pursuiting are widely researched. When decision makers make decisions, he is not risk neutral. In Wang and Webster (2009) [6]'s article, decision space is divided into four parts which are failure, loss, benefit and success. Decision makers will consider the influence of three reference points. In our article, we discuss that decision makers will be influenced by the minimum requirement and the status quo reference point. That is to say, decision makers will divide the profit into three regions. In different areas, decision makers perceive different profits.

Basing on the $M R$ and the $S Q$ reference points, we consider a simple piecewise linear form of value function. The newsvendor has the following function and plot (Figure 2):

$$
V(\pi(x, Q))=\left\{\begin{array}{lc}
\beta_{F} \cdot(\pi-M R)+\beta_{-} M R, & -w Q \leq \pi<M R \\
\beta_{-} \cdot \pi, & M R \leq \pi<S Q \\
\pi, & S Q \leq \pi
\end{array}\right.
$$

We define $\beta_{F}$ and $\beta_{-}$as the coefficient of failure-aversion and the coefficient of loss-aversion. The condition of $\beta_{F}>\beta_{-}>1$ illustrates that the newsvendor has an 


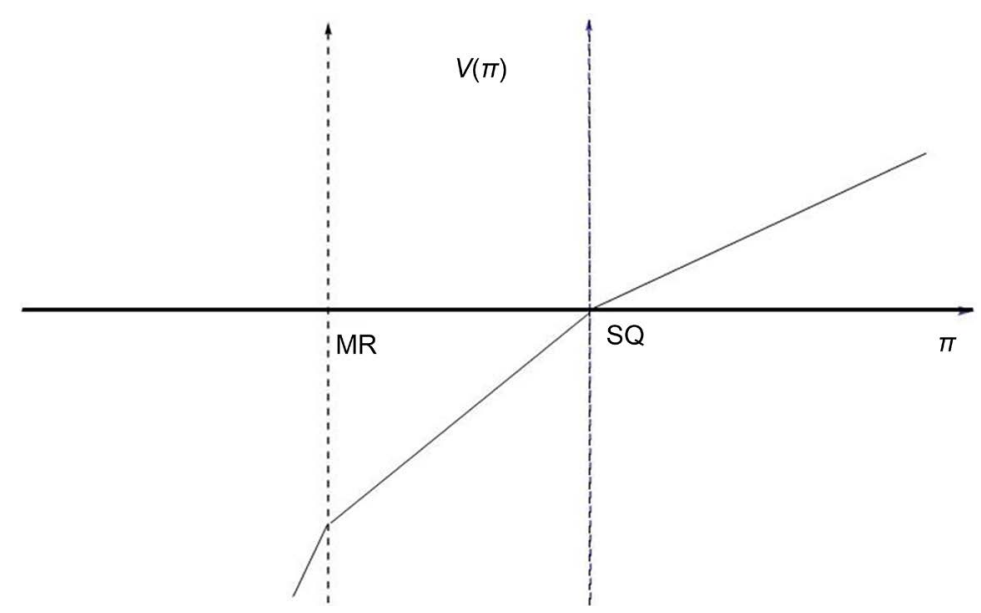

Figure 2. The value function based on $M R$ and $S Q$ reference points.

loss-aversion effect under the status quo reference point and an failure-aversion effect under the minimum requirement reference point. And the degree of failure aversion is greater than the degree of loss aversion depending on the value of the aversion parameters $\beta_{F}$ and $\beta_{-}$. The minimum requirement reference point and status quo reference point are both exogenous. From the article about the status quo reference point, we also normalize the newsvendor's status quo reference point to zero, i.e., $S Q=0$.

For the newsvendor's profit function and value function, we can express his expected utility function as

$$
\begin{aligned}
E(V(\pi(x, Q)))= & \int_{0}^{q_{M}(Q)}\left[\beta_{F} \cdot\left(\pi_{+}-M R\right)+\beta_{-} \cdot M R\right] \cdot f(x) \mathrm{d} x+\int_{q_{M}(Q)}^{q_{S}(Q)}\left[\beta_{-} \cdot \pi_{+}\right] \cdot f(x) \mathrm{d} x \\
& +\int_{q_{S}(Q)}^{Q} \pi_{+} \cdot f(x) \mathrm{d} x+\int_{Q}^{\sup I} \pi_{-} \cdot f(x) \mathrm{d} x
\end{aligned}
$$

The utility function of newsvendor model based on the $M R$ and the $S Q$ reference points above can be rewritten as

$$
\begin{aligned}
E(V(\pi(x, Q)))= & E_{0}(V(\pi(x, Q)))+\left(\beta_{-}-1\right)\left[\int_{0}^{q_{S}(Q)} \pi_{+} \cdot f(x) \mathrm{d} x\right] \\
& +\left(\beta_{F}-\beta_{-}\right)\left[\int_{0}^{q_{M}(Q)}\left(\pi_{+}-M R\right) \cdot f(x) \mathrm{d} x\right]
\end{aligned}
$$

The utility function is the expected profit plus the total expected losses and expected failure losses, biased by the factor of $\left(\beta_{F}-\beta_{-}\right)$and $\left(\beta_{-}-1\right)$. The effect of loss and failure are ordering too much. When we set $\beta_{F}=\beta_{-}=1$, the utility function is the same as the traditional newsvendor model's utility function. This situation is often called as risk neutral.

Theorem 1. For all $Q$ in the definition of the range, $E(V(\pi(x, Q)))$ is concave. And the optimal order quantity $Q_{2}^{*}$ that maximizes $E(V(\pi(x, Q)))$ satisfies the following first-order condition:

$$
-w F\left(Q_{2}^{*}\right)+(p-w) \bar{F}\left(Q_{2}^{*}\right)+\left(\beta_{-}-1\right)\left[-w F\left(q_{S}\left(Q_{2}^{*}\right)\right)\right]+\left(\beta_{F}-\beta_{-}\right)\left[-w F\left(q_{M}\left(Q_{2}^{*}\right)\right)\right]=0
$$

The proof of Theorem is in the Appendix. When we set $\beta_{F}=\beta_{-}=1$, then we can get the traditional newsvendor's utility function. The corresponding first-order condition re- 
duces to $-w F\left(Q_{0}^{*}\right)+(p-w) \bar{F}\left(Q_{0}^{*}\right)=0$. Defining $w F\left(q_{S}\left(Q_{2}^{*}\right)\right)$ as marginal overage loss. For the part of MR reference point, we also define $w F\left(q_{M}\left(Q_{2}^{*}\right)\right)$ as marginal overage failure. It shows that marginal overage loss/failure of a certain order quantity respect to the risk-neutral optimal order quantity. The following proposition will elaborate how to influnce decision bias.

Theorem 2. For any aversion parameters $\beta_{F}>\beta_{-}>1$, there exists $\left(\beta_{-}-1\right)\left[-w F\left(q_{S}\left(Q_{2}^{*}\right)\right)\right]+\left(\beta_{F}-\beta_{-}\right)\left[-w F\left(q_{M}\left(Q_{2}^{*}\right)\right)\right]<0$, then, $Q_{2}^{*}<Q^{*}$.

Theorem 2 illustrates that the newsvendor will generate negative bias which usually show ordering too much, Comparing to the risk-neutral newsvendor. From the theorem 2, we can know that the subject certainly decrease the optimal order quantity with respect to the risk-neutral newsvendor.

Corollary 1. For any $\beta_{F}>\beta_{-}>1$, there exist aversion parameter $\beta_{F}$ and $\beta_{-}$ which make $\left(\beta_{F}-\beta_{-}\right)\left[-w F\left(q_{M}\left(Q_{2}^{*}\right)\right)\right]<\left(\beta_{-}-1\right)\left[-w F\left(q_{S}\left(Q_{2}^{*}\right)\right)\right]$.

As we know, the existence of the $M R$ and $S Q$ reference point will reduce the optimal quantity. But we are not sure the influence degree of MR reference point and $S Q$ reference point. The corollary above expounds the existence of the situation in which the loss of $M R$ part is bigger than the loss of $S Q$ part. In Wang and Webster (2009) [6]'s article, they have been verified the minimum requirement is better than the status quo while decision maker make decisions. That is to say, the first considering condition is the minimum requirement. However, in our article, the happening of this kind of situation depends on the aversion parameters. If the $\beta_{F}$ is infinite, the minimum requirement must be better than the status quo. Although this kind of circumstance must happen, but also exists the status quo is better than the minimum requirement.

For the demand distribution, we choose uniform distribution to verify the correctness of the mathematical results. Specific results can be shown in Figure 3. The value of the parameters are set as follows: $p=2, w=0.8, \beta_{-}=3$ and $M R=-0.3$. Demand is subjected to the uniform distribution of 0 to $200 .\left(\beta_{-}-1\right)\left[-w F\left(q_{S}\left(Q_{2}^{*}\right)\right)\right]$ and $\left(\beta_{F}-\beta_{-}\right)\left[-w F\left(q_{M}\left(Q_{2}^{*}\right)\right)\right]$ refer to the loss of $S Q$ and the loss of MR respectively.

Figure 3 also shows that the newsvendor model based on $M R$ and $S Q$ reference points can well illustrate decision bias. Both of $\left(\beta_{-}-1\right)\left[-w F\left(q_{S}\left(Q_{2}^{*}\right)\right)\right]$ and $\left(\beta_{F}-\beta_{-}\right)\left[-w F\left(q_{M}\left(Q_{2}^{*}\right)\right)\right]$ are negative. They have a intersection point which is about $(8.33,-0.38)$. When $\beta_{F}$ is higher than 8.33 , we can see $\left(\beta_{F}-\beta_{-}\right)\left[-w F\left(q_{M}\left(Q_{2}^{*}\right)\right)\right]<\left(\beta_{-}-1\right)\left[-w F\left(q_{S}\left(Q_{2}^{*}\right)\right)\right]$. Otherwise, we can see the value of $\left(\beta_{-}-1\right)\left[-w F\left(q_{S}\left(Q_{2}^{*}\right)\right)\right]$ is higher than the value of $\left(\beta_{F}-\beta_{-}\right)\left[-w F\left(q_{M}\left(Q_{2}^{*}\right)\right)\right]$. According to the formula in Corollary 1 , when the decision maker very afraid failure, the value of $\beta_{F}$ tend to infinite. The value of $\left(\beta_{-}-1\right)\left[-w F\left(q_{S}\left(Q_{2}^{*}\right)\right)\right]$ is constant smaller than the value of $\left(\beta_{F}-\beta_{-}\right)\left[-w F\left(q_{M}\left(Q_{2}^{*}\right)\right)\right]$. 


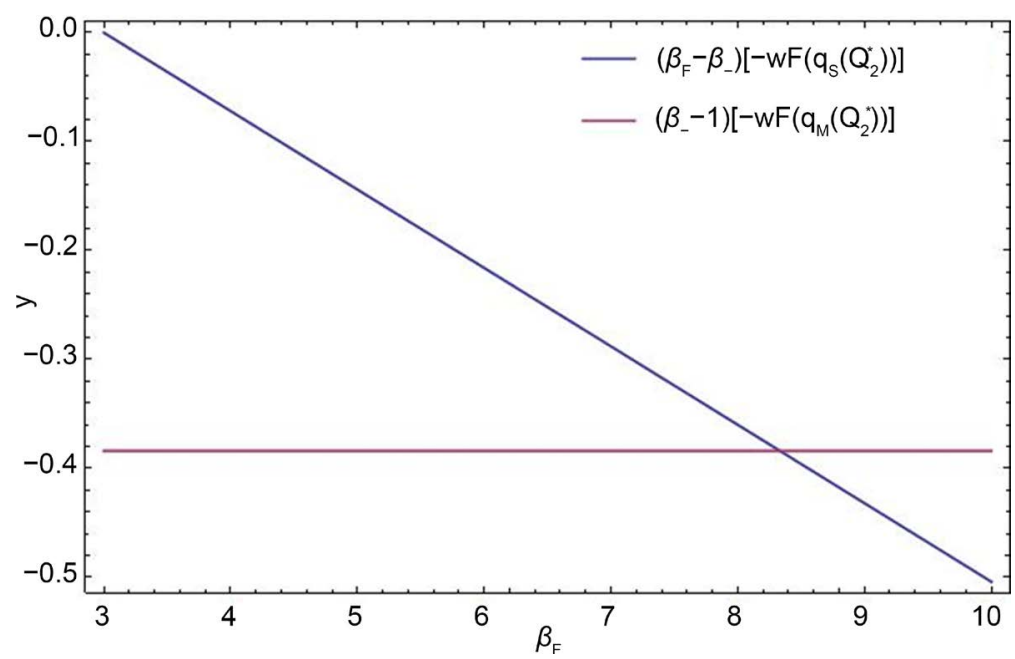

Figure 3. The value of decision bias, in uniform distribution, with $p=2, w=0.8, \beta_{-}=3$ and $M R=-0.3$.

\section{Comparative Statics}

In our model, we use mathematical analysis to investigate other parameters's influence on the newsvendor's optimal order quantity. Then, we apply uniform distribution to test our analysis. Combining with mathematical analysis and numerical analysis to illustrate our view.

Theorem 3. For parameter changes, optimal order quantity $Q_{2}^{*}$ has following characters.

1) $Q_{2}^{*}$ is increasing in $\mathrm{p}$;

2) $Q_{2}^{*}$ is decreasing in $\mathrm{w}, \mathrm{MR}, \beta_{-}$and $\beta_{F}$;

We also get some conclusions about ordering cost $\mathrm{w}$ and retail price $p . M R$ as a exogenous reference point is discussed in the following part. For the risk-neutral newsvendor model, the single $S Q$ reference point newsvendor model and the newsvendor model based on $M R$ and $S Q$ reference points, optimal order quantity is increasing in retail price $\mathrm{p}$ while decreasing in ordering cost w. Theorem (3) (see Appendix for proof) identify optimal order quantity's changing direction. Adding in $M R$ and $S Q$ reference points makes the discussion about ordering quantity more complex.

In order to better understand the impact of parameters, we use some numerical examples to illustrate the decision bias. Generally speaking, uniform is widely used in various extended newsvendor models. For uniform distribution, we assume mean demand $D / 2=100$. For loss aversion parameter and failure aversion parameter, we choose different $\beta_{F}$ and $\beta_{-}$.

Figure 4(a) and Figure 4(b) show that the newsvendor model based on $M R$ and $S Q$ reference points can well illustrate decision bias with retail price $\mathrm{p}$ and wholesale price w changing. Figure 4(c), Figure 4(d) and Figure 4(e) illustrate psychological parameters's influence on optimal quantity. In Figure $4(\mathrm{a}), Q_{2}^{*}$ is increasing in retail price $p$. That is to say, overage loss is getting small when the retail price $p$ increases. Because the optimal quantity on $S Q$ and $M R$ reference points is less than that of the risk neutral 


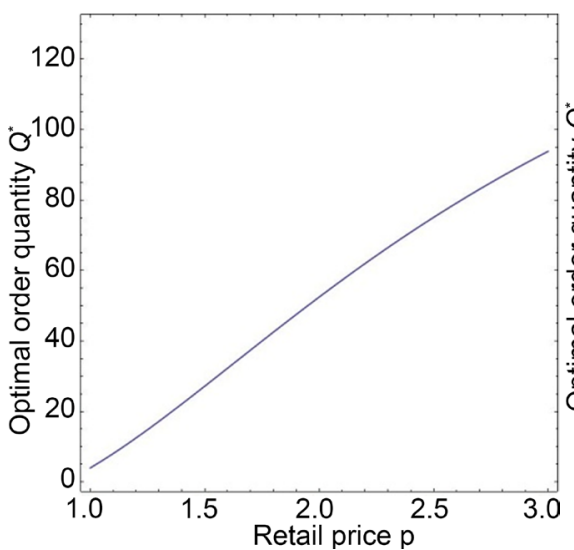

(a)

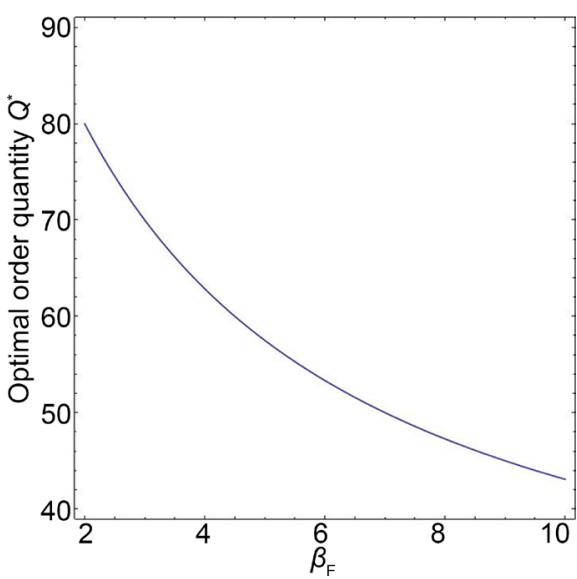

(c)

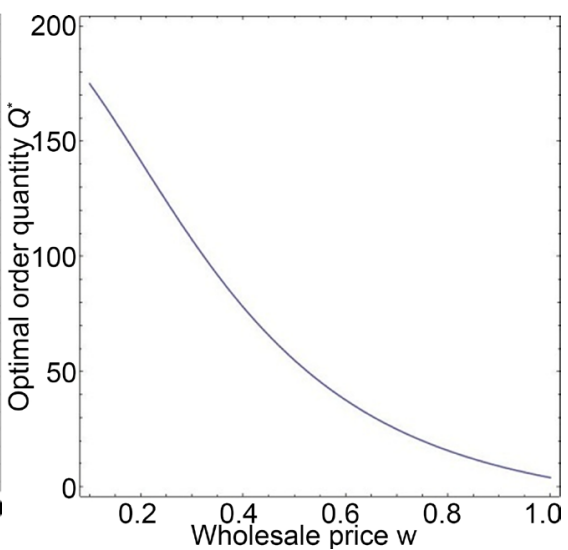

(b)

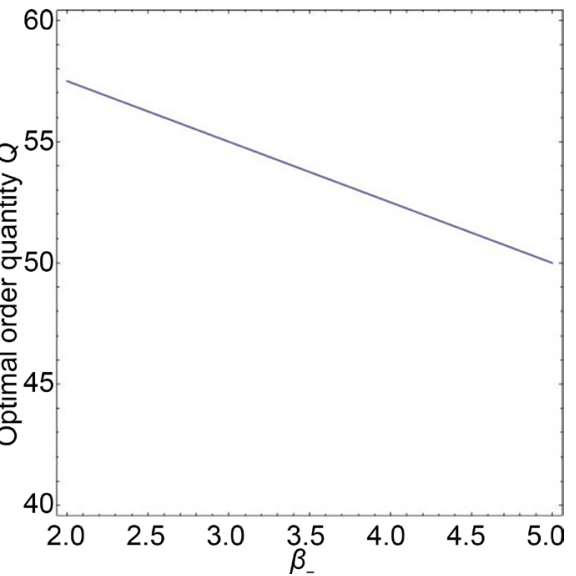

(d)

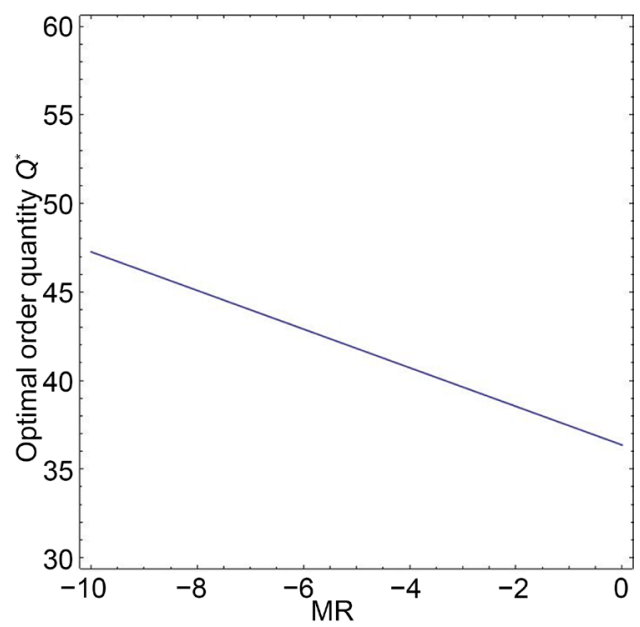

(e)

Figure 4. (a) The influence of retail price $p$, in uniform distribution, with $w=1, \beta_{-}=3, \beta_{F}=5$ and $M R=-10$; (b) The influence of wholesale price $w$, in uniform distribution, with $p=1$, $\beta_{-}=3, \beta_{F}=5$ and $M R=-10$; (c) The influence of $\beta_{F}$ in uniform distribution, with $p=1, w=$ $0.5, \beta_{-}=2$ and $M R=-10$; (d) The influenceof $\beta_{-}$, in uniform distribution, with $p=1, w=$ $0.5, \beta_{F}=5$ and $M R=-10$; (e) The influence of $M R$, in uniform distribution, with $p=1, w=1$, $\beta_{-}=3$ and $\beta_{F}=8$. 
in Therom 1. Raising retail price p will drive up the optimal quantity on $S Q$ and $M R$ reference points. Thus they can make up for the loss of the psychological influence. Likewise, $Q_{2}^{*}$ is decreasing in wholesale price $w$ in Figure 4(b). Reducing wholesale price $w$ will drive up the optimal quantity on SQ and MR reference points.

In our model, the optimal quantity $Q_{2}^{*}$ decreases with aversion parameters $\beta_{F}$ and $\beta_{-}$increasing in Figure 4(c) and Figure 4(d). And the value of MR increases, while the optimal quantity $Q_{2}^{*}$ decreases in Figure $4(\mathrm{e})$. Similarly, Theorem 1 atates adding $S Q$ and $M R$ reference points will reduce the optimal quantity with respect to the risk neutral. Due to overage loss, the existence of the aversion parameters will expand this loss. For the $M R$ reference point, we can know that when $M R$ is infinitesimal, the decision maker cannot perceive the existence of MR. With the rise of $M R$, when the decision maker can perceive the existence of $M R$, he will decrease his optimal quantity for avoiding failure. In Figure 4(e), we can see the optimal quantity is the biggest at the lowest $M R$. The status quo is usually set to a fixed value of 0 . We don't need to discuss its changing status.

Comparing to the traditional newsvendor model, which shows optimal order quantity always decreasing in $w$ and increasing in $p$, the newsvendor model based on $M R$ and $S Q$ reference points gives more conditions. Recent articles confirm the impact of changes in the $M R$ reference point on the optimal order quantity.

\section{Conclusions}

Part 3 shows, based on these results, we can understand the $M R$ as an important reference point in explaining the importance of the decision on the bias, to further expand the research of multi-reference points. In random demand of purchasing decision, the decision makers of actual order behavior will be influenced by reference points. This article based on the minimum requirement and status quo reference points build the decision-making behavior of newsvendor model. It explains the mechanism of newsvendor decision-making deviation from the perspective of multiple reference points. Through the analysis of the mathematical model and numerical examples, it verifies the deviation and changing direction.

From the theorem and numerical examples of part 4, we explain, in the current situation based on $M R$ and $S Q$ reference points, how parameters influence the optimal quantity. And we further explain how the minimum requirement and the status quo affect the order decision simultaneously. As previously described to, Agrawal and Seshadri (2000) [4] find decision bias for a risk-averse newsvendor. Schweitzer and Cachon (2000) [5] use newsvendor experiments to test the decision bias and propose the existence of a pull-to-center effect. For multiple reference points, Diener (2009) [13] and Ordonez, Connolly et al. (2000) [14] propose the muti-reference point should be applied to explain the decision bias. Researching on the newsvendor model of MR and SQ reference points can better explain how a plurality of parameters influence on ordering decision.

In all, this paper mainly discusses the influence of $M R$ and $S Q$ reference point of the 
newsvendor's order decision under stochastic demand. We combine the loss aversion, crossing failure, and use overage failure cost and overage loss cost to analyze the decision bias in the newsvendor problem. Our conclusions show that, adding $M R$ and $S Q$ reference point must decrease the optimal order quantity, which is that the newsvendor order less items. And with the $M R$ reference point increasing, the optimal order quantity will decrease. This situation may illustrate that, when the $M R$ reference point is infinitesimal, the newsvendor do not change his order decision due to feeling nothing about $M R$ reference point. When the $M R$ reference point reach the degree of newsvendor's feel, a little quantity may decrease. Then with the $M R$ reference point continue increasing, the optimal order quantity will continue decreasing. Finally, when the $M R$ reference point reaches the maximum point, the newsvendor will order nothing. No matter how much the newsvendor orders, he feels negative profit. So ordering is not wise. With wholesale price and retail price increasing, the optimal order quantity will be more or less in some conditions, which is the same as some papers. In another case, the conclusion is opposite.

We also conclude some future research directions about this paper. Setting endogenous reference point will be interesting to see the newsvendor's psychological perception. Goal reference point can also be added into this model to analyze decision bias. Newsvendor's decision is no longer a single cycle, but multi-cycle. Around the discussion of the reference point, we can better learn the true impact of decision-making. Psychology experiments and empirical articles have confirmed the existence of the reference points (see [11] [14]). Mathematical analysis of how the multiple effects reference point affects the ordering decision will be a good research topic.

\section{References}

[1] Arrow, K.J., Harris, T. and Marschak, J. (1951) Optimal Inventory Policy. Econometrica: Journal of the Econometric Society, 250-272. http://dx.doi.org/10.2307/1906813

[2] Khouja, M. (1999) The Single-Period (News-Vendor) Problem: Literature Review and Suggestions for Future Research. Omega, 27, 537-553. http://dx.doi.org/10.1016/S0305-0483(99)00017-1

[3] Eeckhoudt, L., Gollier, C. and Schlesinger, H. (1995) The Risk-Averse (and Prudent) Newsboy. Management Science, 41, 786-794. http://dx.doi.org/10.1287/mnsc.41.5.786

[4] Agrawal, V. and Seshadri, S. (2000) Impact of Uncertainty and Risk Aversion on Price and Order Quantity in the Newsvendor Problem. Manufacturing \& Service Operations Management, 2, 410-423. http://dx.doi.org/10.1287/msom.2.4.410.12339

[5] Schweitzer, M.E. and Cachon, G.P. (2000) Decision Bias in the Newsvendor Problem with a Known Demand Distribution: Experimental Evidence. Management Science, 46, 404-420. http://dx.doi.org/10.1287/mnsc.46.3.404.12070

[6] Wang, C.X. and Webster, S. (2009) The Loss-Averse Newsvendor Problem. Omega, 37, 93105. http://dx.doi.org/10.1016/j.omega.2006.08.003

[7] Bernoulli, D. (1954) Exposition of a New Theory on the Measurement of Risk. Econometrica: Journal of the Econometric Society, 23-36. http://dx.doi.org/10.2307/1909829

[8] Neumann, J.V. and Morgenstern, O. (1944) Theory of Games and Economic Behavior. Princeton University Press, Princeton. 
[9] Tversky, A. and Kahneman, D. (1992) Advances in Prospect Theory: Cumulative Representation of Uncertainty. Journal of Risk and Uncertainty, 5, 297-323. http://dx.doi.org/10.1007/BF00122574

[10] Kahneman, D. and Tversky, A. (1979) Prospect Theory: An Analysis of Decision under Risk. Econometrica: Journal of the Econometric Society, 263-291. http://dx.doi.org/10.2307/1914185

[11] Wang, X.T. and Johnson, J.G. (2012) A Tri-Reference Point Theory of Decision Making under Risk. Journal of Experimental Psychology: General, 141, 743. http://dx.doi.org/10.1037/a0027415

[12] Kacelnik, A. and Bateson, M. (1997) Risk-Sensitivity: Crossroads for Theories of DecisionMaking. Trends in Cognitive Sciences, 1, 304-309. http://dx.doi.org/10.1016/S1364-6613(97)01093-0

[13] Diener, E., Sandvik, E. and Pavot, W. (2009) Happiness Is the Frequency, Not the Intensity, of Positive versus Negative Affect. 213-231.

[14] Ordóñez, L.D., Connolly, T. and Coughlan, R. (2000) Multiple Reference Points in Satisfaction and Fairness Assessment. Journal of Behavioral Decision Making, 13, 329. http://dx.doi.org/10.1002/1099-0771(200007/09)13:3<329::AID-BDM356>3.3.CO;2-H

[15] Rosch, E. (1975) Cognitive Reference Points. Cognitive Psychology, 7, 532-547. http://dx.doi.org/10.1016/0010-0285(75)90021-3

[16] Su, X. (2008) Bounded Rationality in Newsvendor Models. Manufacturing \& Service Operations Management, 10, 566-589. http://dx.doi.org/10.1287/msom.1070.0200

[17] Nagarajan, M. and Shechter, S. (2013) Prospect Theory and the Newsvendor Problem. Management Science, 60, 1057-1062. http://dx.doi.org/10.1287/mnsc.2013.1804

[18] Zhao, X. and Geng, W. (2014) A Note on "Prospect Theory and the Newsvendor Problem". Journal of the Operations Research Society of China, 3, 89-94. http://dx.doi.org/10.1007/s40305-015-0072-4

[19] Uppari, B.S. and Hasija, S. (2014) On the Consistency between Prospect Theory and the Newsvendor Pull-to-Center Effect.

[20] Allais, M. (1953) Le comportement de l'homme rationnel devant le risque: Critique des postulats et axiomes de l'école américaine. Econometrica, 21, 503-546. http://dx.doi.org/10.2307/1907921

[21] Ellsberg, D. (1961) Risk, Ambiguity, and the Savage Axioms. Quarterly Journal of Economics, 75, 643-669. http://dx.doi.org/10.2307/1884324

[22] Schmidt, U., Starmer, C. and Sugden, R. (2008) Third-Generation Prospect Theory. Journal of Risk and Uncertainty, 36, 203-223. http://dx.doi.org/10.1007/s11166-008-9040-2

[23] Stephens, D.W. and Krebs, J.R. (1986) Foraging Theory. Princeton University Press, Princeton.

[24] Sullivan, K. and Kida, T. (1995) The Effect of Multiple Reference Points and Prior Gains and Losses on Managers' Risky Decision Making. Organizational Behavior and Human Decision Processes, 64, 76-83. http://dx.doi.org/10.1006/obhd.1995.1091

[25] Yates, J.F. and Stone, E.R. (1992) The Risk Construct.

[26] Real, L. and Caraco, T. (1986) Risk and Foraging in Stochastic Environments. Annual Review of Ecology and Systematics, 17, 371-390.

http://dx.doi.org/10.1146/annurev.es.17.110186.002103 


\section{Appendix}

Proof of Theorem 1.

(1) If $q_{M}\left(Q_{2}^{*}\right)>0$, we can get the expected utility Function (6)

$$
\begin{aligned}
E(V(\pi(x, Q)))= & E_{0}(V(\pi(x, Q)))+\left(\beta_{-}-1\right)\left[\int_{0}^{q_{S}(Q)} \pi_{-} \cdot f(x) \mathrm{d} x\right] \\
& +\left(\beta_{F}-\beta_{-}\right)\left[\int_{0}^{q_{M}(Q)}\left(\pi_{-}-M R\right) \cdot f(x) \mathrm{d} x\right]
\end{aligned}
$$

Since $Q$ is continuous and derivative, we take the first derivative of Function (6) and get:

$$
\begin{aligned}
\frac{\mathrm{d} E(V(\pi(x, Q)))}{\mathrm{d} Q}= & -w F(Q)+(p-w) \bar{F}(Q)+\left(\beta_{-}-1\right)\left[-w F\left(q_{S}(Q)\right)\right] \\
& +\left(\beta_{F}-\beta_{-}\right)\left[-w F\left(q_{M}(Q)\right)\right]
\end{aligned}
$$

After taking second derivative of $E(V(\pi(x, Q)))$ with respect to $Q$, we get

$$
\frac{\mathrm{d} E^{2}(V(\pi(x, Q)))}{\mathrm{d} Q^{2}}=-p f(Q)-\left(\beta_{-}-1\right)\left[\frac{w^{2}}{p} f\left(q_{S}(Q)\right)\right]-\left(\beta_{F}-\beta_{-}\right)\left[\frac{w^{2}}{p} f\left(q_{M}(Q)\right)\right]
$$

For all $Q \in I, \frac{\mathrm{d} E^{2}(V(\pi(x, Q)))}{\mathrm{d} Q^{2}}$ is negative. Hence, if $q_{M}\left(Q_{2}^{*}\right)>0$, then there exists a unique optimal $Q_{2}^{*}$ that satisfies the first-order condition (6).

(2) If $q_{M}\left(Q_{2}^{*}\right)<0$, we can get the expected utility Function (7)

$$
E(V(\pi(x, Q)))=E_{0}(V(\pi(x, Q)))+\left(\beta_{-}-1\right)\left[\int_{0}^{q_{S}(Q)} \pi_{-} \cdot f(x) \mathrm{d} x\right]
$$

Since $Q$ is continuous and derivative, we take the first derivative of Function (7) and get:

$$
\frac{\mathrm{d} E(V(\pi(x, Q)))}{\mathrm{d} Q}=-w F(Q)+(p-w) \bar{F}(Q)+\left(\beta_{-}-1\right)\left[-w F\left(q_{S}(Q)\right)\right]
$$

After taking second derivative of $E(V(\pi(x, Q)))$ with respect to $Q$, we get

$$
\frac{\mathrm{d} E^{2}(V(\pi(x, Q)))}{\mathrm{d} Q^{2}}=-p f(Q)-\left(\beta_{-}-1\right)\left[\frac{w^{2}}{p} f\left(q_{S}(Q)\right)\right]
$$

For all $Q \in I, \frac{\mathrm{d} E^{2}(V(\pi(x, Q)))}{\mathrm{d} Q^{2}}$ isis negative. There exists a unique optimal $Q_{2}^{*}$ that satisfies the first-order condition.

Proof of Theorem 3.

$$
\frac{\mathrm{d} Q_{2}^{*}}{\mathrm{~d} p}=\frac{\frac{\mathrm{d} E^{2}(V(\pi(x, Q)))}{\mathrm{d} Q \mathrm{~d} p}}{-\frac{\mathrm{d} E^{2}(V(\pi(x, Q)))}{\mathrm{d} Q^{2}}}=\frac{\bar{F}\left(Q_{2}^{*}\right)+\left(\beta_{-}-1\right) \frac{w(M R+w Q) f\left(q_{M}\left(Q_{2}^{*}\right)\right)}{p^{2}}+\left(\beta_{F}-\beta_{-}\right) \frac{w^{2} Q f\left(q_{M}\left(Q_{2}^{*}\right)\right)}{p^{2}}}{-\frac{\mathrm{d} E_{2 M S}^{2}\left(V\left(\pi_{r}\right)\right)}{\mathrm{d} Q^{2}}}>0
$$




$$
\begin{aligned}
& \frac{\mathrm{d} Q_{2}^{*}}{\mathrm{~d} w}=\frac{\frac{\mathrm{d} E^{2}(V(\pi(x, Q)))}{\mathrm{d} Q \mathrm{~d} w}}{-\frac{\mathrm{d} E^{2}(V(\pi(x, Q)))}{\mathrm{d} Q^{2}}} \\
& =\frac{-1-\left(\beta_{-}-1\right)\left[F\left(q_{S}\left(Q_{2}^{*}\right)\right)+\frac{w Q}{p} f\left(q_{S}\left(Q_{2}^{*}\right)\right)\right]-\left(\beta_{F}-\beta_{-}\right)\left[F\left(q_{M}\left(Q_{2}^{*}\right)\right)+\frac{w Q}{p} f\left(q_{M}\left(Q_{2}^{*}\right)\right)\right]}{-\frac{\mathrm{d} E_{2 M S}^{2}\left(V\left(\pi_{r}\right)\right)}{\mathrm{d} Q^{2}}}<0 \\
& \frac{\mathrm{d} Q_{2}^{*}}{\mathrm{~d} M R}=\frac{\frac{\mathrm{d} E^{2}(V(\pi(x, Q)))}{\mathrm{d} Q \mathrm{~d} M R}}{-\frac{\mathrm{d} E^{2}(V(\pi(x, Q)))}{\mathrm{d} Q^{2}}}=\frac{-\left(\beta_{F}-\beta_{-}\right) \frac{W f\left(q_{M}\left(Q_{2}^{*}\right)\right)}{P}}{-\frac{\mathrm{d} E_{2 M S}^{2}\left(V\left(\pi_{r}\right)\right)}{\mathrm{d} Q^{2}}}<0 \\
& \frac{\mathrm{d} Q_{2}^{*}}{\mathrm{~d} \beta_{F}}=\frac{\frac{\mathrm{d} E^{2}(V(\pi(x, Q)))}{\mathrm{d} Q \mathrm{~d} \beta_{F}}}{-\frac{\mathrm{d} E^{2}(V(\pi(x, Q)))}{\mathrm{d} Q^{2}}}=\frac{-w F\left(q_{M}\left(Q_{2}^{*}\right)\right)}{-\frac{\mathrm{d} E_{2 M S}^{2}\left(V\left(\pi_{r}\right)\right)}{\mathrm{d} Q^{2}}}<0 \\
& \frac{\mathrm{d} Q_{2}^{*}}{\mathrm{~d} \beta_{-}}=\frac{\frac{\mathrm{d} E^{2}(V(\pi(x, Q)))}{\mathrm{d} Q \mathrm{~d} \beta_{-}}}{-\frac{\mathrm{d} E^{2}(V(\pi(x, Q)))}{\mathrm{d} Q^{2}}}=\frac{-w\left[F\left(q_{S}\left(Q_{2}^{*}\right)\right)-F\left(q_{M}\left(Q_{2}^{*}\right)\right)\right]}{-\frac{\mathrm{d} E_{2 M S}^{2}\left(V\left(\pi_{r}\right)\right)}{\mathrm{d} Q^{2}}}<0
\end{aligned}
$$

Submit or recommend next manuscript to SCIRP and we will provide best service for you:

Accepting pre-submission inquiries through Email, Facebook, LinkedIn, Twitter, etc.

A wide selection of journals (inclusive of 9 subjects, more than 200 journals)

Providing 24-hour high-quality service

User-friendly online submission system

Fair and swift peer-review system

Efficient typesetting and proofreading procedure

Display of the result of downloads and visits, as well as the number of cited articles

Maximum dissemination of your research work

Submit your manuscript at: http://papersubmission.scirp.org/

Or contact jss@scirp.org 